\title{
Manifestaciones en la cavidad bucal y en la cara asociadas a la COVID-19
}

\section{Manifestations in the Oral Cavity and Face Associated with COVID-19}

Recibido: 29 Marzo 2021 | Aceptado: 11 Mayo 2021

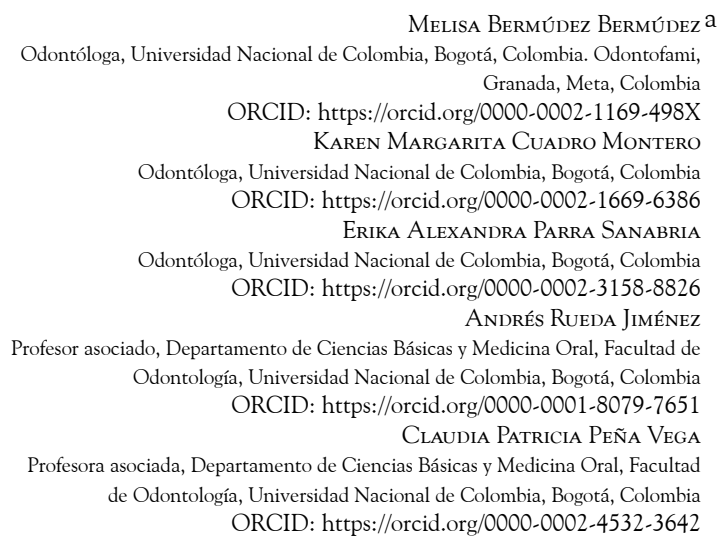

Melisa Bermúdez Bermúdeza

Odontóloga, Universidad Nacional de Colombia, Bogotá, Colombia. Odontofami, Granada, Meta, Colombia

ORCID: https://orcid.org/0000-0002-1169-498X Karen Margarita Cuadro Montero Odontóloga, Universidad Nacional de Colombia, Bogotá, Colombia ORCID: https://orcid.org/0000-0002-1669-6386 Erika Alexandra Parra Sanabria Odontóloga, Universidad Nacional de Colombia, Bogotá, Colombia ORCID: https://orcid.org/0000-0002-3158-8826 Andrés Rueda Jiménez ORCID: https://orcid.org/0000-0001-8079-7651 Claudia Patricia Peña Vega Profesora asociada, Departamento de Ciencias Básicas y Medicina Oral, Facultad de Odontología, Universidad Nacional de Colombia, Bogotá, Colombia ORCID: https://orcid.org/0000-0002-4532-3642

a Autora de correspondencia: mbermudezb@unal.edu.co

Cómo citar: Bermúdez Bermúdez M, Cuadro Montero KM, Parra Sanabria EA, Rueda Jiménez A, Peña Vega CP. Manifestaciones en la cavidad bucal y en la cara asociadas a la COVID-19. Univ. Med. 2021;62 (3). ht tps://doi.org/10.11144/Javeriana.umed62-3.mcbc

\section{RESUMEN}

Objetivo: Describir y analizar los reportes de casos y estudios publicados, en los que se presente una posible relación entre la COVID-19 y lesiones mucocutáneas en la cavidad oral y la cara. Metodología: Revisión en las bases de datos de PubMed, Google Académico, ProQuest y Medline Complete. Se seleccionaron 56 artículos que reportan manifestaciones mucocutáneas en la cavidad oral y la cara asociadas a la COVID-19. Resultados: Se encontraron 91 casos con manifestaciones mucocutáneas en la cavidad oral y la cara, asociadas a la COVID-19. En el $18 \%$ de los pacientes hubo lesiones tanto faciales como orales. Las mejillas fueron las zonas más afectadas (con un $16 \%$ ), y de las lesiones orales, el sitio más afectado fue la lengua (26\%). Conclusión: De acuerdo con los hallazgos encontrados, la presencia de manifestaciones mucocutáneas en la cara y la cavidad oral están asociadas con la infección por SARSCoV-2. Es de esperarse que los tejidos con mayor expresión de la ECA2 y TMPRSS2 sean más susceptibles a la infección por el SARS-CoV-2 y, por consiguiente, presenten algún tipo de lesión. Dentro de los tejidos orales con mayor expresión de dichas moléculas se describió la lengua, lo que favorecería mayor prevalencia de lesiones.

Palabras clave

COVID-19; facial; mucosa; manifestaciones orales.

\section{ABSTRACT}

Objective: To describe and analyze case reports and published studies in which a possible relationship between COVID-19 and mucocutaneous lesions in the oral cavity and face is presented. Methodology: A review was performed in Pubmed, Google Scholar, ProQuest, and Medline Complete databases, 56 articles were selected reporting mucocutaneous manifestations in oral cavity and face associated to COVID-19. Results: 
We found 91 reported cases with mucocutaneous manifestations in oral cavity and face, associated with COVID-19. Eighteen percent of the patients presented lesions at both facial and oral levels. The cheeks were the most affected areas representing $16 \%$ and of oral lesions the most affected site was the tongue with $26 \%$. Conclusion: According to the findings, the presence of mucocutaneous manifestations in face and oral cavity are associated with SARS-CoV-2 infection. It is expected that tissues with higher expression of ECA2 and TMPRSS2 are more susceptible to infection by SARS-CoV-2 and therefore present some type of lesion. Among the oral tissues with higher expression of these molecules, the tongue was described, which would favor a higher prevalence of lesions.

Keywords

COVID-19; facial; mucous membrane; oral manifestations.

\section{Introducción}

El SARS-CoV-2 infecta las células huésped al unirse al receptor transmembrana de la enzima convertidora de angiotensina 2 (ECA2) por medio de la proteína estructural S (Spike), presente en la superficie viral. Dicha proteína se compone de dos subunidades: la subunidad S1, que se encarga de unirse al receptor de la ECA2 de la célula huésped (1), y la S2, que se encarga de fusionar la membrana del virus con la de la célula huésped $(2,3)$, por lo que dicha enzima tiene un papel fundamental en la infectividad del SARS-CoV-2. Esto permite concluir que las células que expresan ECA2 son más susceptibles de ser infectadas por el virus (4). Después del ingreso del virus a la célula huésped, se acelera su replicación y diseminación, y con ello la infección de otras células que expresan ECA2, como las células pulmonares y las capilares. El resultado es el aumento y la amplificación de la respuesta inflamatoria, capaz de llevar a un estadio severo, en el que se presenta una hipercitocinemia proinflamatoria y una respuesta sistémica no controlada. Ello puede deteriorar los órganos afectados, como los pulmones, y generar el síndrome de dificultad respiratoria aguda $(5,6)$.

Se han descrito varias vías por medio de las cuales el SARS-CoV-2 puede infectar las células humanas. Dentro de estas se menciona la TMPRSS2, una molécula del hospedero que actúa como una proteasa importante en el proceso de invasión viral en la célula hospedera, ya que activa la proteína $S$ y le facilita su unión con la ECA2 $(3,7)$. Otra molécula descrita es la furina, una enzima celular responsable de activar el proceso por el que el SARS-CoV-2 se une a los receptores de membrana de la célula del huésped mediante su proteína de unión Spike (8).

Las manifestaciones clínicas producidas por la COVID-19 se han descrito como heterogéneas, ya que varían desde síntomas leves o casi inexistentes hasta complicaciones fatales, como insuficiencia respiratoria, choque séptico e insuficiencia multiorgánica. Los síntomas iniciales también han sido variables, y en la mayoría de los pacientes, el inicio de los síntomas ha incluido fiebre y alteraciones respiratorias (9).

En cuanto a la sintomatología extrapulmonar, la COVID-19 se ha asociado con manifestaciones orales y faciales. Dentro de los casos publicados de manifestaciones orales se han descrito lesiones en la mucosa oral, úlceras, petequias, ampollas, parotiditis $(10,11)$, trastornos quimiosensibles como disgeusia y anosmia $(12,13)$ - , enantemas virales, gingivitis ulceronecrotizante, entre otras (14). Algunos autores también han mencionado que los medicamentos administrados durante el tratamiento de la infección por SARS-CoV-2, el estrés y los factores sistémicos de base podrían tener un efecto causal de dichas manifestaciones; sin embargo, todavía está limitado el nivel de evidencia (15-17).

Tanto la expresión de la ECA2 como de la TMPRSS2 se han descrito en varios órganos del cuerpo. Estudios como los realizados por Xu et al. (18) han enfocado su atención en la expresión de la ECA2 en los tejidos de la cavidad oral y han encontrado una alta expresión en el dorso lingual; también han mencionado su expresión en las papilas gustativas y en los tejidos gingivales. Otros hallazgos expuestos por Sakaguchi et al. (8) indican una mayor expresión de la ECA2 y de la TMPRSS2 en las células escamosas epiteliales descamadas que están presentes en la mucosa lingual, así como una marcada expresión en las papilas gustativas y en algunas zonas del epitelio gingival; sin embargo, los autores sugieren más estudios para especificar la distribución de las distintas moléculas expresadas que participan en 
el proceso de infección del SARS-CoV-2 (tabla 1).

\section{Tabla 1}

Expresión de marcadores determinantes de la infección por SARSCoV2 en la cavidad oral y piel

\begin{tabular}{|c|c|c|c|c|}
\hline \multicolumn{2}{|c|}{ Tipo de tejido } & \multirow{2}{*}{\begin{tabular}{|c|c}
$\begin{array}{c}\text { Nivel de } \\
\text { expresión } \\
\text { ECA2 }\end{array}$ \\
No se menciona
\end{tabular}} & \multirow{2}{*}{\begin{tabular}{|c|c|}
$\begin{array}{c}\text { Nivel de } \\
\text { expresión } \\
\text { TMPRSS2 }\end{array}$ \\
+ \\
\end{tabular}} & \multirow{2}{*}{$\begin{array}{cc}\begin{array}{c}\text { Nivel de } \\
\text { expresión } \\
\text { Furina }\end{array} \\
- & \\
\end{array}$} \\
\hline $\begin{array}{l}\text { Epitelio } \\
\text { escamoso }\end{array}$ & \begin{tabular}{|l}
$\begin{array}{l}\text { Superficie } \\
\text { epitelial }\end{array}$ \\
\end{tabular} & & & \\
\hline lingual & Capa córnea & $+(*)$ & \pm & - \\
\hline & \begin{tabular}{|l|}
$\begin{array}{l}\text { Capa } \\
\text { espinosa }\end{array}$ \\
\end{tabular} & + & + & + \\
\hline & \begin{tabular}{|l|} 
Capa basal \\
\end{tabular} & + & $\pm(* *)$ & ++ \\
\hline & \begin{tabular}{|l|} 
Células \\
descamadas
\end{tabular} & H+ & +H & - \\
\hline \multirow[t]{3}{*}{$\begin{array}{l}\text { Papilas } \\
\text { gustativas }\end{array}$} & \begin{tabular}{|l|}
$\begin{array}{l}\text { Células } \\
\text { epiteliales }\end{array}$ \\
\end{tabular} & + & No se menciona & No se menciona \\
\hline & \begin{tabular}{|l} 
Células de las \\
papilas \\
gustativas
\end{tabular} & No se menciona & +++ & No se menciona \\
\hline & $\begin{array}{l}\text { Capas } \\
\text { inferiores de } \\
\text { las papilas }\end{array}$ & No se menciona & No se menciona & + \\
\hline \multirow{4}{*}{$\begin{array}{l}\text { Epitelio } \\
\text { escamoso } \\
\text { gingival }\end{array}$} & \begin{tabular}{|l|}
$\begin{array}{l}\text { Superficie } \\
\text { epitelial }\end{array}$ \\
\end{tabular} & - & + & - \\
\hline & Capa córnea & - & \pm & - \\
\hline & \begin{tabular}{|l|} 
Capa \\
espinosa
\end{tabular} & + & + & + \\
\hline & \begin{tabular}{|l|} 
Capa basal \\
\end{tabular} & + & \pm & ++ \\
\hline \multirow[t]{2}{*}{$\begin{array}{l}\text { Epitelio } \\
\text { gingival }\end{array}$} & $\begin{array}{l}\begin{array}{l}\text { Epitelio bucal } \\
\text { surcular }\end{array} \\
\end{array}$ & +++ & +++ & $++1+$ \\
\hline & $\begin{array}{l}\begin{array}{l}\text { Epitelio bucal } \\
\text { gingival }\end{array} \\
\end{array}$ & ++ & ++ & ++ \\
\hline
\end{tabular}

$$
\begin{gathered}
\pm \text { expresión baja; }+ \text { expresión positiva; } \\
++ \text { expresión altamente positiva; }+ \\
++ \text { expresión fuertemente positiva. } \\
* \text { Inconsistente y esporádicamente } \\
\text { observada como una región de } \\
\text { señalización relativamente fuerte. } \\
* * \text { Mayoritariamente negativa. } \\
\text { Fuente: adaptado de }(8) .
\end{gathered}
$$

La presente revisión busca brindar información sobre la relación entre la COVID-19 y las posibles manifestaciones mucocutáneas de cara y cavidad oral, por medio de la descripción y análisis de reportes de casos y estudios publicados, en los que se presente esta posible asociación.

\section{Métodos}

Se revisó la literatura sobre el tema en las bases de datos PubMed, Google Académico, ProQuest y Medline Complete para artículos publicados hasta el 15 de enero del 2021, en los cuales se hubieran documentado manifestaciones mucocutáneas que involucraran la cara y la cavidad oral asociadas con la presencia de infección por SARS-CoV-2. Se rastrearon artículos con texto completo publicados en español o inglés, que se leyeron y seleccionaron de acuerdo con los criterios de inclusión. Dentro de los términos de búsqueda empleados se utilizaron las palabras COVID-19, facial, mucous membrane . oral manifestations, relacionándolos con ayuda del conector booleano AND. Se incluyeron presentaciones de casos, cartas al editor y comunicaciones breves, en las que se describieran pacientes diagnosticados con la COVID-19 y que hubieran presentado manifestaciones mucocutáneas que afectaran la cavidad oral y la cara. Se excluyeron los artículos que no cumplieran con los criterios de búsqueda, que estuvieran incompletos o que mencionaran lesiones cutáneas que no afectaban la cavidad oral o la región facial.

La consulta inicial en las 4 bases de datos mencionadas arrojó un total de 5099 artículos, de los cuales se revisó el título o el contenido y se seleccionaron los que incluyeran los términos de búsqueda relacionados con el objetivo de esta pesquisa. De estos se excluyeron 5043 artículos, que no reportaban casos clínicos o que no cumplían los criterios relacionados con la búsqueda. Así, quedaron un total de 56 artículos que cumplían con el objetivo de la revisión.

Se analizaron los artículos seleccionados y se evaluó su contenido, teniendo en cuenta los datos clínicos que se proporcionaban: sexo, edad y antecedentes médicos de los pacientes, diagnóstico confirmado o sospechoso de la COVID-19, manifestaciones orales y faciales y posible etiología.

\section{Resultados}

Se rastrearon 91 pacientes, con una mayor prevalencia de lesiones en el sexo femenino, comparado con el sexo masculino, con un 55 $\%$ y $45 \%$ de los casos, respectivamente. La edad promedio fue de 43 años. El $93 \%$ de los casos fueron confirmados para la COVID-19 mediante las pruebas de PCR o hisopado nasal. En cuanto a las lesiones observadas, el $18 \%$ de los pacientes presentaron manifestaciones tanto faciales como orales (anexo). Respecto a las condiciones sistémicas de los pacientes, el 28,2 
\% reportaba algún tipo de antecedente sistémico como hipertensión, diabetes, asma, obesidad, entre otros; mientras que el $71,8 \%$ restante no tenía antecedentes.

\section{Lesiones orales}

De todas las lesiones de la cavidad oral, los sitios más afectados fueron la lengua, con el $26 \%$; seguida de los labios, con el $21 \%$, y el paladar (paladar duro o blando), con el 20\%. Los sitios con menor afectación fueron la mucosa bucal y la encía, con el $8 \%$ cada una; seguidas de la orofaringe y la comisura labial, con el $6 \%$ y el 5 $\%$, respectivamente, y el piso de boca y la zona retromolar, con el 3\%. El 3\% restante fueron reportadas en la cavidad oral sin describir un sitio específico de afectación (anexo).

En cuanto a los tipos de lesiones, se encontró que eran muy variadas. La mayoría correspondieron al grupo de úlceras y erosiones, con un $16 \%$, y estomatitis (estomatitis aftosa, aftas y aftas menores), con el $16 \%$. También se describieron máculas y enantema con el $7 \%$ cada una; eritema y necrosis (necrosis superficial y necrosis hemorrágica), con el $6 \%$ cada una; queilitis o queilitis comisural, gingivitis (gingivitis ulcerativa necrotizante, gingivitis descamativa) y petequias, con el $5 \%$ cada una; seguidas de lesiones por virus del herpes simple (VHS), con el $4 \%$; candidiasis, con el $3 \%$, y angina bullosa hemorrágica y depapilación lingual, con el $2 \%$ cada una. Finalmente, los tipos de lesiones que presentaron solo un caso fueron incluidas en el grupo de "otros", el cual correspondió al $16 \%$ de las lesiones (anexo).

\section{Lesiones cutáneas faciales}

Para la clasificación de las lesiones cutáneas, se tuvo en cuenta la propuesta por Marzano et al. (19), en 2020. De todas las lesiones faciales que se presentaron, la mayoría de las publicaciones no especificaron la ubicación exacta, por lo que un $49 \%$ se describen en la cara. Dentro de las lesiones cuya ubicación fue especificada, las mejillas fueron las zonas más afectadas, con un $16 \%$ de las lesiones. De estas, todas fueron asociadas con presión (ventilación mecánica o posición prono). Otras zonas menos afectadas fueron el mentón, la zona malar, la sien, entre otros, que representaron un $35 \%(20,21)$ (anexo).

En cuanto a la clasificación de las lesiones reportadas, la mayoría fue de tipo exantema u otras lesiones inflamatorias (lesiones urticariales, máculopapulares - eritematosas confluentes y papulovesiculares) con un $49 \%$, seguidas de las lesiones tipo vasculíticas (patrón purpúrico "vasculítico") con el $32 \%$ y por último las lesiones posiblemente relacionadas con hipersensibilidad medicamentosa que representaron un $20 \%$.

Dentro de las lesiones, se encontró que las más frecuentes fueron las lesiones vasculíticas del tipo úlceras, con un $32 \%$ (de las cuales el $85 \%$ correspondió a úlceras púrpura/necróticas asociadas a daño vascular por presión), seguidas de las lesiones exantematosas/inflamatorias que incluyen los tipos mácula (papular eritematosa, eritematosa, papular, pustulosa y urticarial), con el $27 \%$; edema/eritema, con el $17 \%$; eritema multiforme y otros (urticaria/vesicular y petequias), con el $10 \%$ cada uno. Por último, las que presentaron menor porcentaje fueron las lesiones exantematosas/inflamatorias papulovesiculares causadas por VHS, con un 5 $\%$. Es importante mencionar que los porcentajes dados han sido redondeados.

\section{Discusión}

Los mecanismos fisiopatológicos de las lesiones orales y cutáneas aún no se han descrito con claridad; sin embargo, como posibles causas se han mencionado una respuesta inmune exagerada, la activación del sistema complemento y un daño en la microvasculatura (19). Por otro lado, la interacción entre el receptor ECA2 y el SARS-CoV-2 podría alterar el revestimiento epitelial de las glándulas salivales y los queratinocitos y provocar lesiones como úlceras en la cavidad oral (22). También se ha encontrado que en pacientes mayores o 
inmunosuprimidos con infección por el SARS$\mathrm{CoV}-2$ las lesiones orales tuvieron presentaciones más graves y generalizadas (22).

\section{Lesiones orales}

\section{Úlceras y erosiones}

Las úlceras en la cavidad oral pueden estar relacionadas con distintos factores etiológicos, como infecciones, alteraciones inmunes, traumatismos o neoplasias (23). Se encontró que es posible que las úlceras se presenten de formas irregulares o definidas, asintomáticas o dolorosas y con lesiones únicas o múltiples. Los sitios más frecuentes en los que se reporta la aparición de estas lesiones son: paladar, labios, comisuras, lengua, mucosa bucal, piso de la boca y zona retromolar $(10,15,20,22,24-33)$ (anexo).

Algunos autores asocian este tipo de lesiones con la presencia de procesos virales, ya que la resolución de dichas lesiones en la cavidad oral suele ocurrir simultáneamente con la resolución de la infección viral $(22,24,27,28,32)$. También se ha descrito como factor causal la presencia de vasculitis inducida por una reacción inflamatoria asociada a la COVID-19 (25). Otros autores atribuyen estas lesiones al estado de inmunosupresión de los pacientes y al estrés de la estancia hospitalaria prolongada $(15,31)$.

En el caso de pacientes que han estado en posición decúbito prono durante el tratamiento de neumonía y síndrome respiratorio agudo severo, causados por la COVID-19, se ha reportado la aparición de úlceras por la posible hipoxemia, lesiones en la microvasculatura y trombosis, que son causales de úlceras por presión (20). Otro factor al que se le ha atribuido la aparición de úlceras es la administración de medicamentos como la hidroxicloroquina o el oseltamivir $(26,27,29)$, que han sido causa de lesiones cutáneas como eritema multiforme, síndrome de StevenJohnson y necrólisis epidérmica tóxica $(34,35)$. Las úlceras orales también se han encontrado en pacientes con la COVID-19, diagnosticados con enfermedad similar a Kawasaki (30). En el caso de las erosiones, se atribuye como factor etiológico el daño viral directo a mucosas y tejido vascular, ya que estos interactúan con el SARSCoV-2 al expresar el receptor de unión ECA2 (10) (anexo).

\section{Estomatitis aftosa, aftas y aftas menores}

Comúnmente, las lesiones aftosas en la cavidad oral se asocian con diferentes factores desencadenantes, como traumatismos, tabaquismo, infecciones, enfermedades reumatológicas, deficiencias nutricionales, medicamentos, entre otros $(9,36)$. Pueden presentarse con zonas necróticas (22). En estudios en los que se relacionan con la presencia de infección por la COVID-19 se ha encontrado que afectan distintas zonas de la cavidad oral, como la mucosa labial, el dorso, los bordes laterales y vientre lingual, el paladar duro, el pilar amigdalino, la mucosa bucal y unión mucogingival; pueden variar desde lesiones únicas a lesiones múltiples, generalmente dolorosas (22,36-39) (anexo).

En los casos documentados de pacientes con la COVID-19 se ha atribuido la aparición de lesiones tipo aftas a exantemas inducidas por la acción viral (40), a una posible reactivación del VHS (22), a una sobreinfección por cándida (41) o una dermatosis neutrofílica febril aguda, generalmente conocida como síndrome de Sweet (SS), aunque ha sido considerado por los autores como un caso poco frecuente, ya que por lo general las lesiones orales son hallazgos raros en el SS (39).

Se ha postulado que estas lesiones se relacionan con efectos provocados por la acción del virus, más que por otros factores, como la reacción a medicamentos, debido a que en muchos casos las lesiones se resolvieron conforme mejoraban los síntomas generales causados por el virus, sin suspender los medicamentos $(22,41)$. En algunas circunstancias, las lesiones orales aparecieron en el momento de evidenciarse alteraciones en el gusto y el olfato (22); mientras que en otro caso las lesiones aparecieron en un 
paciente asintomático (41). Las aftas menores tendieron a afectar con mayor frecuencia a los pacientes más jóvenes, en tanto que las lesiones más severas y generalizadas afectaron con más frecuencia a los pacientes mayores con estados severos de la enfermedad $(22,36)$.

Dentro de los mecanismos fisiopatológicos se ha descrito que las lesiones pueden estar relacionadas con la alta expresión de la ECA2 en los tejidos de la lengua y las glándulas salivares, lo que aumenta la susceptibilidad de dichos a tejidos a ser infectados por el virus y, por consiguiente, a ser lesionados por la acción inflamatoria $(8,22)$. También se ha mencionado la acción del FNT- $\alpha$, presente en la hipercitocinemia, desencadenada por la presencia del virus, lo que llevaría a los neutrófilos a atacar la mucosa oral (36). Finalmente, se ha propuesto que la respuesta exagerada de los neutrófilos ante la infección por el virus puede desencadenar las manifestaciones del SS (39) (anexo).

\section{Máculas}

Las máculas se describieron como lesiones rojizas, púrpuras o blanquecinas que afectaron principalmente la lengua, el paladar duro y los labios. Todas las lesiones documentadas fueron asintomáticas e incluyeron desde lesiones únicas con márgenes difusos hasta lesiones múltiples irregulares dispersas $(28,41-43)$. La mayoría de los autores atribuye estas lesiones a la acción del virus en el organismo, más que a la reacción medicamentosa, alteraciones vasculares o alteraciones inmunológicas aisladas $(28,42,43)$. Se sugiere que pueden estar relacionadas con un patrón exantémico inducido por la acción inflamatoria con el virus (42) o con un trastorno vascular trombótico inducido por la acción de mediadores del sistema complemento en las paredes microvasculares, lo cual genera un daño en las células endoteliales y activa factores de coagulación. Ello lleva a una trombosis microvascular con la consecuente alteración cutánea (4344) (anexo).

\section{Eritema}

Las lesiones eritematosas pueden ser sintomáticas o asintomáticas. Por lo general, se describe una lesión única. Se ha reportado su aparición en la lengua, el paladar duro, la orofaringe y las amígdalas $(25,27,45-50)$. La aparición de este tipo de lesiones se ha asociado con una posible vasculitis (25) que podría ser inducida por inflamación vascular (51), exantema viral $(45,46,50)$, enfermedad similar a la de Kawasaki (47), deterioro de la salud sistémica e inmunosupresión (48), tratamientos farmacológicos para la COVID-19 (27) o una posible reacción de hipersensibilidad de la mucosa a la presencia del SARS-CoV-2 en el epitelio (49) (anexo).

\section{Enantemas}

Los enantemas pueden encontrarse con petequias, máculas, pápulas o vesículas en la cavidad oral. Su presencia suele asociarse fuertemente con una etiología viral, sobre todo cuando se observa un patrón petequial (42). $\mathrm{Su}$ presentación es múltiple en la mayoría de los casos y no se reporta sintomatología $(30,42,45)$. En la presente revisión, algunos autores atribuyen la aparición de este tipo de lesiones, principalmente, a un exantema viral $(42,45)$, aunque en un paciente infectado con la COVID-19 también se encontró asociación con una enfermedad similar a la de Kawasaki (30) (anexo).

\section{Necrosis}

Las lesiones tipo necrosis fueron encontradas en labios y lengua, en presentación múltiple o única, con sintomatología o sin esta $(22,52,53)$. Las lesiones necróticas aparecen con mayor frecuencia en ancianos y en pacientes graves o inmunosuprimidos (22) (anexo). En cuanto a la causa, se evidenció que su desaparición se daba en paralelo a la resolución de la COVID-19, por lo que algunos reportes mencionan que 
estas manifestaciones podrían estar directamente relacionadas con la infección por el SARS-CoV-2 (22). Otra posible explicación se le atribuyó a la presencia de microclusiones dérmicas asociadas con hemoglobinuria paroxística nocturna que, en conjunto con el daño endotelial inducido por la COVID-19, dio como resultado una necrosis hemorrágica exagerada (52). Finalmente, se encontró la aparición de áreas necróticas que podrían ser producto de hipoxemia inducida, ya sea por disminución en la oxigenación o por presión de un dispositivo para la ventilación mecánica, en combinación con una afectación vascular o disminución del flujo sanguíneo, lo que produciría isquemia en periodos prolongados (53) (anexo).

\section{Queilitis}

Las lesiones queilíticas se describieron como fisuras, lesiones escamosas o erosiones en las comisuras labiales. Pueden presentarse de forma uni o bilateral, generalmente son dolorosas y, en algunos casos, están asociadas a cándida $(38,54,55)$. Dentro de los reportes se han descrito fisuras en las comisuras labiales, junto con sensación de boca seca, disgeusia y ageusia; en estos casos se sugiere que estas lesiones se atribuyan a estados de inmunosupresión o estrés (38). Otros casos describieron lesiones queilíticas en niños de 3 y 9 años, junto con conjuntivitis, glositis, estomatitis y exantema generalizado, por lo que los autores concluyeron que podrían ser casos de enfermedad de Kawasaki, posiblemente desencadenada por la infección por la COVID-19 $(54,55)$.

Adicionalmente, se han descrito casos de queilitis con erosiones gingivales difusas, costras labiales hemorrágicas, conjuntivitis y múltiples erosiones cutáneas en forma de diana, por lo que los autores los clasificaron como eritema multiforme, posiblemente asociado a la COVID-19 (55) (anexo).

\section{Petequias}

Pocos autores indicaron la presencia de petequias. Estas se ubicaron en el paladar duro, en las encías, en los labios o de forma generalizada en la cavidad oral $(10,41,45,56)$. Entre los síntomas asociados con la presencia de petequias en la cavidad oral se mencionó exantema macular generalizado y costras hemorrágicas en las superficies internas de los labios (10), eritema en la orofaringe y pústulas en el paladar blando (45) y signos de coinfección por cándida (41) (anexo).

En cuanto a su relación con la severidad de la COVID-19, algunos casos se dieron en pacientes con síntomas graves que requirieron hospitalización $(10,56)$; mientras que en otros casos se presentaron en pacientes asintomáticos o con síntomas leves $(41,45)$. Dentro de las posibles causas se ha mencionado trombocitopenia asociada a hipercitocinemia y estados hiperinflamatorios, consecuencia de la infección por el SARS-COV-2, favorecida o potenciada por la acción de algunos analgésicos como ibuprofeno (41) o algunos antibióticos, como las cefalosporinas, administrados para el manejo de los síntomas de la COVID-19 (10) (anexo).

\section{Gingivitis}

Las lesiones que afectaron el tejido gingival incluyeron gingivitis, gingivitis ulcerativa necrotizante y gingivitis descamativa. Todas estas lesiones fueron múltiples y dolorosas. Su mejoría ocurrió en simultáneo con la recuperación de la sintomatología de la COVID-19 $(14,24,57)$ (anexo).

En cuanto a la etiología de la gingivitis ulcerativa necrotizante, se atribuye su aparición a una coinfección bacteriana en simultáneo con la COVID-19 (14). En el caso de la gingivitis descamativa, se propone un exantema viral provocado por la acción del SARS-CoV-2 (24), ya que se ha observado la aparición de lesiones tipo ampolla, úlceras, aftas, gingivoestomatitis herpética, etc., resultado de la acción viral (58). 
En los casos de gingivitis se le atribuye un origen multifactorial, favorecido por la presencia de placa bacteriana, debido a una deficiencia en las medidas de higiene oral (57) (anexo).

\section{Virus del herpes simple}

Las lesiones por VHS se han descrito en la mucosa bucal, labios y lengua. No se informó sintomatología y los pacientes no tuvieron afectación sistémica. Este tipo de lesiones se presentaron como prurito o vesículas orales $(59,60)$. Dentro de las causas atribuidas a esta manifestación se menciona una posible reactivación retrógrada del virus varicela zóster o herpes simple recurrente, potenciadas por la reacción inflamatoria asociada a la COVID-19 $(59,61,62)$. También se ha atribuido la aparición de vesículas orales como una manifestación cutánea de la COVID-19 (60) (anexo).

\section{Candidiasis}

La candidiasis seudomembranosa oral se ha descrito como una lesión en parches o placas blancas localizadas o dispersas, con apariencia membranosa, que puede ser dolorosa o asociarse a una sensación de ardor en la zona afectada. En los casos vinculados con la COVID-19, se han ubicado en el dorso lingual y en el paladar $(38,41,63)$. Solo en un caso se reportó una enfermedad de base, el hipotiroidismo (63), pero no se describieron condiciones sistémicas adicionales (anexo).

La Candida albicans hace parte de la flora normal de la cavidad oral y se ha descrito que puede generar infecciones oportunistas ante procesos inflamatorios, xerostomía, utilización de antibióticos de amplio espectro (azitromicina, linezolid, ceftriaxona) o alteraciones de la respuesta inmune; incluso se ha mencionado que el sexo femenino tiene una leve tendencia a presentar más riesgo de infecciones oportunistas $(33,38,41,63-65)$ (anexo).

\section{Angina bullosa hemorrágica}

Se caracteriza por la aparición repentina de una o varias ampollas tensas, de contenido hemático, en la mucosa oral o faríngea. La localización más frecuente es en la unión del paladar blando con el paladar duro, pero también pueden afectar el dorso o las zonas laterales de la lengua (66).

En los pacientes con infección por el SARS-CoV-2, las lesiones tipo angina bullosa hemorrágica fueron encontradas en la lengua y el paladar blando, respectivamente, sin sintomatología. Los dos casos publicados se encontraron en pacientes sin afectación sistémica. Probablemente, estos tipos de lesiones están asociadas de forma directa con la COVID-19 (43) (anexo).

\section{Depapilación lingual}

Los casos de depapilación lingual en pacientes con la COVID-19 han presentado disgeusia, anosmia, sensación de ardor y boca seca como síntomas asociados. No había relación con enfermedades de base. Se observó que la depapilación apareció en todo el dorso lingual o en los bordes únicamente $(38,49)$. Dentro de las posibles causas, se propuso una relación con cierto de grado de inmunosupresión asociada a la infección por el SARS-CoV-2 (38) o una mucositis oral generada por hipersensibilidad a la presencia del SARS-CoV-2 en el epitelio afectado. En este último caso, se planteó la posibilidad de describir estas lesiones como trastornos oportunistas secundarios asociados a la respuesta inmune en contra del virus. Sin embargo, se resaltó que no se debe descartar que sean lesiones primarias por acción directa del virus sobre los tejidos (49) (anexo).

\section{Otras lesiones orales}

Dentro de las lesiones menos frecuentes se describieron grietas y fisuras labiales, papilas linguales prominentes, estomatitis y glositis. En todos estos casos, las lesiones se relacionaron 
con la enfermedad de Kawasaki asociada a la COVID-19, ya que aparecieron en conjunto con conjuntivitis y exantemas $(47,54,55,67,68)$. A pesar de no conocerse una fisiopatología exacta de la enfermedad de Kawasaki, dentro de las posibles causas se describió la hipercitocinemia, desencadenada en repuesta al SARS-CoV-2 (54) (anexo).

También se describieron ampollas principalmente en los labios, además de lesiones similares a estomatitis herpética recurrente, las cuales son consideradas comunes en los procesos virales $(24,58)$. En el caso de las ampollas, se clasificaron como lesiones relacionadas con eritema multiforme asociado a la COVID-19; sin embargo, es necesario realizar más estudios para determinar los mecanismos que generan estas lesiones (24).

Igualmente, se describieron lesiones nodulares (fibromas) en el labio inferior, clasificadas como una posible lesión reactiva por el uso de dispositivos para la intubación (27); lesiones tipo pápula en el paladar, asociadas a un trastorno vascular inducido por la respuesta inflamatoria contra el SARS-CoV-2 (43), y pigmentaciones melánicas gingivales transitorias, las cuales se atribuyeron a la estimulación de los melanocitos gingivales por el proceso inflamatorio y a la predisposición genética (41) (anexo).

\section{Lesiones faciales}

Para analizar las lesiones faciales relacionadas con la COVID-19 encontradas en el presente estudio, se tomó como base la clasificación publicada por Marzano et al. (19), en la cual se agruparon las lesiones según sus patrones y características clínicas. Basado en lo anterior, se clasificaron las lesiones cutáneas, según el tipo exantémico/inflamatorio o vasculopático/ vasculítico.

\section{Erupciones exantematosas/inflamatorias}

Erupción urticarial. Las lesiones urticariales se describen como ronchas o placas rojas, sobreelevadas, de aparición repentina, que pueden o no estar relacionadas con prurito $(37,69)$. En la mayoría de los casos se han asociado con pacientes de la COVID-19 que están bajo manejo con medicamentos debido a la gravedad de los síntomas (9). En cuanto a su distribución, principalmente, suelen afectar el tronco, pero también las extremidades y la cabeza, excluyendo en la mayoría de los casos las palmas y las plantas de los pies $(9,70-76)$ (anexo).

Erupción confluente eritematosa maculopapular morbiliforme. Las lesiones maculopapulares se caracterizan por la presencia de máculas eritematosas que pueden estar cubiertas por pápulas pequeñas o placas grandes, asociarse a descamación y ser confluentes, lo que favorece que dichas lesiones se confundan con pitiriasis rosada $(37,77-80)$. Se atribuyen a la presencia de partículas virales en los vasos sanguíneos de la piel, lo cual puede desencadenar la formación de pápulas debido a la diapédesis de linfocitos que se acumulan y producen inflamación $(52,81,82)$. Se ha documentado que las lesiones maculopapulares afectan principalmente las extremidades y el tronco $(70,76,83,84)$, pero también pueden afectar la cara $(73,85-89)$ y los talones (69) (anexo).

Exantemas. Estas lesiones se caracterizan por la presencia de vesículas llenas de líquido que se encuentran sobre una base eritematosa (37), pustulosis exantémicas (90), edema facial (91), erupción folicular urticarial (92), etc. Se han observado lesiones papulovesiculares generalizadas en el cuerpo, con enantema petequial en el paladar y lesiones eritematosas en la cara, atribuidas a un posible exantema viral (42,92-94). También se ha atribuido la aparición de lesiones exantematosas en la cara y el cuello a una posible reacción adversa a medicamentos utilizados en el tratamiento de la COVID-19 como lopinavir, ritonavir, hidroxicloroquina y ceftriaxona. Esta respuesta puede verse potenciada por una respuesta inmune contra el virus $(90,91,95-97)$ (anexo). 


\section{Lesiones vasculopáticas/vasculiticas}

La aparición de estas lesiones se ha relacionado con la presión prolongada en los casos en los que se ha requerido ventilación mecánica. Los autores han propuesto que dicha presión provoca hipoxemia en los tejidos afectados y genera lesiones microvasculares y trombosis, lo que da como resultado úlceras faciales (20). También se han reportado lesiones similares a la enfermedad de Kawasaki, las cuales se han descrito como una vasculitis leucocitoclástica, donde pueden evidenciarse necrosis de la epidermis $(68,98)$ (anexo).

Relación de lesiones orales con la expresión de marcadores como ECA2 y TMPRSS2

Estudios histológicos han demostrado que aquellos tejidos con una mayor expresión de los marcadores ECA2 y TMPRSS2 son más susceptibles de ser infectados por el SARS-CoV-2 y, por tanto, presentar algún tipo de lesión. Entre los tejidos orales en los que se ha descrito una mayor expresión de estos marcadores se mencionó la lengua, en especial las células descamadas superficiales. Aunque estas últimas son infectadas con mayor facilidad por el SARSCoV-2, aún no es claro si pueden favorecer la infección de células de estratos más profundos del epitelio lingual e inducir, a su vez, la inflamación y manifestación de lesiones linguales. Por ello, se requieren más estudios para determinar los mecanismos que puedan generar algún tipo de lesión oral (8) (tabla 1).

En la presente revisión se encontró una alta frecuencia de lesiones linguales, como depapilación $(38,49)$, papilas linguales prominentes $(50,68)$, glositis $(54,55)$, úlceras (25), lengua geográfica (27), aftas (22, 41), entre otras, lo cual podría explicarse con la alta expresión de los marcadores ECA2 y TMPRSS2; sin embargo, todavía siguen siendo necesarios más estudios para determinar con claridad los mecanismos fisiopatológicos que inducen dichas lesiones.

\section{Conclusiones}

De acuerdo con los hallazgos encontrados, la presencia de manifestaciones mucocutáneas en la cara y la cavidad oral está asociada con la infección por SARS-CoV-2. Es de esperarse que los tejidos con mayor expresión de la ECA2 y TMPRSS2 sean más susceptibles a la infección por el SARS-CoV-2 y, por consiguiente, presenten algún tipo de lesión. Dentro de los tejidos orales con mayor expresión de dichas moléculas se describió la lengua y sus células descamadas, lo que favorecería una mayor prevalencia de lesiones, como se evidenció con los casos reportados. Por otro lado, deben considerarse factores, como la severidad de la COVID-19, tratamientos farmacológicos, ventilación mecánica, coinfecciones y estado inmunológico del paciente, que pueden contribuir a un mayor riesgo para estas lesiones.

\section{Conflictos de intereses}

Los autores no manifiestan presentar conflictos de intereses.

\section{Referencias}

1. Zou X, Chen K, Zou J, Han P, Hao J, Han Z. Single-cell RNA-seq data analysis on the receptor ACE2 expression reveals the potential risk of different human organs vulnerable to 2019-nCoV infection. Front Med. 2020;14(2):185-92.

2. Yuki K, Fujiogi M, Koutsogiannaki S. COVID-19 pathophysiology: a review. Clin Immunol. 2020 Jun 1;215:1-7.

3. Wiersinga WJ, Rhodes A, Cheng AC, Peacock SJ, Prescott HC. Pathophysiology, transmission, diagnosis, and treatment of coronavirus disease 2019 (COVID-19): a Review. JAMA. 2020 Jul 10;324(8):782-93. htt ps://doi.org/10.1001/jama.2020.12839 
4. Zhang H, Kang Z, Gong H, Xu D, Wang J, Li Z, et al. The digestive system is a potential route of $2019-\mathrm{nCov}$ infection: a bioinformatics analysis based on single-cell transcriptomes. . 2020 Jan 1. https://doi.org/10.1101/202 0.01 .30 .927806

5. Li X, Geng M, Peng Y, Meng L, Lu S. Molecular immune pathogenesis and diagnosis of COVID-19. J Pharm Anal. 2020 Apr 1;10(2):102-8.

6. Cao W, Li T. COVID-19: towards understanding of pathogenesis. Cell Res. 2020 May 1;30(5):367-9.

7. Ou X, Liu Y, Lei X, Li P, Mi $\mathrm{D}$, Ren L, et al. Characterization of spike glycoprotein of SARS-CoV-2 on virus entry and its immune cross-reactivity with SARS-CoV. Nat Commun. 2020;11(1):1-12. https://doi .org/10.1038/s41467-020-15562-9

8. Sakaguchi W, Kubota N, Shimizu T, Saruta J, Fuchida S, Kawata A, et al. Existence of SARS-CoV-2 entry molecules in the oral cavity. Int J Mol Sci. 2020;21(17):1-16.

9. Galván Casas C, Català A, Carretero Hernández G, Rodríguez-Jiménez P, Fernández Nieto D, Rodríguez-Villa Lario A, et al. Classification of the cutaneous manifestations of COVID-19: a rapid prospective nationwide consensus study in Spain with 375 cases. $\mathrm{Br} J$ Dermatol. 2020;183:71-7.

10. Ciccarese G, Drago F, Boatti M, Porro A, Muzic SI, Parodi A. Oral erosions and petechiae during SARSCoV-2 infection. J Med Virol. 2020. ht tps://doi.org/10.1002/jmv.26221

11. Sinadinos A, Shelswell J. Oral ulceration and blistering in patients with COVID-19. Evid Based Dent. 2020;21(2):49.

12. Vinayachandran D, Balasubramanian S. Is gustatory impairment the first report of an oral manifestation in COVID-19? Oral Dis. 2020;1-5.

13. Biadsee AA, Biadsee AA, Kassem F, Dagan O, Masarwa S, Ormianer Z. Olfactory and oral manifestations of COVID-19: sex-related symptoms -a potential pathway to early diagnosis. Otolaryngol Head Neck Surg. 2020;163(4):722-8. https://doi.or g/10.1177/0194599820934380

14. Patel J, Woolley J. Necrotizing periodontal disease: Oral manifestation of COVID-19. Oral Dis. 2020;0-2.

15. Ansari R, Gheitani M, Heidari FF, Heidari FF. Oral cavity lesions as a manifestation of the novel virus (COVID-19): a letter-to-editor. Oral Dis. 2020 Jun 8.

16. Al-Khatib A. Oral manifestations in COVID-19 patients. Oral Dis. 2020;1-2.

17. das Chagas e Silva de Carvalho LF, Kitakawa D, Cabral LAG. Oral lesions of herpes zoster in COVID-19 patients or truly associated to the disease? Oral Dis. 2020 Jun 10;1-2.

18. Xu H, Zhong L, Deng J, Peng J, Dan $\mathrm{H}$, Zeng $\mathrm{X}$, et al. High expression of ACE2 receptor of 2019-nCoV on the epithelial cells of oral mucosa. Int J Oral Sci. 2020;12(8). https://doi.org/ 10.1038/s41368-020-0074-x

19. Marzano A V., Cassano N, Genovese G, Moltrasio C, Vena GA. Cutaneous manifestations in patients with COVID-19: a preliminary review of an emerging issue. $\mathrm{Br} \mathrm{J}$ Dermatol. 2020;183(3):431-42.

20. Perrillat A, Foletti JM, Lacagne AS, Guyot L, Graillon N. Facial pressure ulcers in COVID-19 patients undergoing prone positioning: How to prevent an underestimated epidemic? J Stomatol oral Maxillofac Surg. 2020 Jun 18;1-3. 
21. Karagounis TK, Shaw KS, Caplan A, Lo Sicco K, Femia AN. Acrofacial purpura and necrotic ulcerations in COVID-19: a case series from New York City. Int J Dermatol. 2020;59(11):1419-22.

22. Brandão TB, Gueiros LA, Melo TS, Prado-Ribeiro AC, Nesrallah ACFA, Prado GVB, et al. Oral lesions in patients with SARS-CoV-2 infection: could the oral cavity be a target organ? Oral Surg Oral Med Oral Pathol Oral Radiol. 2021;131(2):e45-51. https://do i.org/10.1016/j.oooo.2020.07.014

23. Fitzpatrick SG, Cohen DM, Clark AN. Ulcerated lesions of the oral mucosa: clinical and histologic review. Head Neck Pathol. 2019;13(1):91-102. https://doi.org/10. 1007/s12105-018-0981-8

24. Carreras Presas MC, Amaro Sánchez J, López Sánchez AF, Jané Salas E, Somacarrera Pérez ML. Oral vesiculobullous lesions associated with SARS-CoV-2 infection. Oral Dis. 2021;27 Suppl 3:710-2. https://doi.org/ 10.1111/odi.13382

25. Chaux-Bodard A-G, Deneuve S, Desoutter A. Oral manifestation of Covid-19 as an inaugural symptom? J Oral Med Oral Surg. 2020;26(18):1.

26. Sakaida T, Tanimoto I, Matsubara A, Nakamura M, Morita A. Unique skin manifestations of COVID-19: Is drug eruption specific to COVID-19? J Dermatol Sci. 99(1):62-4. https://doi.o rg/10.1016/j.jdermsci.2020.05.002

27. Santos JA dos, Normando AGC, Silva RLC da, Paula RM De, Cembranel AC, Santos-Silva AR, et al. Oral mucosal lesions in a COVID-19 patient: new signs or secondary manifestations? Int J Infect Dis. 2020 Jun;97.

28. Soares C, Carvalho R, Carvalho $\mathrm{K}$, Carvalho M, Almeida O. Letter to editor: oral lesions in a patient with Covid-19. Med Oral Patol Oral y Cir Bucal. 2020;25(4):563-4.

29. Demirbaş A, Elmas ÖF, Atasoy M, Türsen Ü, Lotti T. A case of erythema multiforme major in a patient with COVID 19: The role of corticosteroid treatment. Dermatol Ther. 2020 Nov;33(6):e13899. https:// doi.org/10.1111/dth.13899

30. Chérif MY, de Filette JMK, André S, Kamgang P, Richert B, Clevenbergh P. Coronavirus disease 2019-related Kawasaki-like disease in an adult: A case report. JAAD Case Reports. 2020;6(8):780-2.

31. Kämmerer $\mathrm{T}$, Walch J, Flaig M, French LE. COVID-19-associated herpetic gingivostomatitis. Clin Exp Dermatol. 2021;46(1):174-6.

32. Bezerra TM, Feitosa SG, Carneiro DTO, Costa FWG, Pires FR, Pereira KMA. Oral lesions in COVID-19 infection: Is long-term follow-up important in the affected patients? Oral Dis. 2020 Oct:1-2.

33. Iranmanesh B, Aflatoonian M, Khalili M, Amiri R. Oral manifestations of COVID-19 disease: a review article. 2021 Jan;34(1):e14578. https://doi.org/10.1111/dth.14578

34. Abou Assalie N, Durcan R, Durcan L, Petri MA. Hydroxychloroquineinduced erythema multiforme. J Clin Rheumatol. 2017;23(2):127-8.

35. Zuo W, Wen L, Li J, Mei D, Fu Q, Zhang B. Oseltamivir induced Stevens - Johnson syndrome/ toxic epidermal necrolysiscase report. Medicine (Baltimore). 2019 May;98(19):e15553. https://doi.o rg/10.1097/MD.0000000000015553

36. Domínguez-Santas M, DíazGuimaraens B, Fernández-Nieto D, Jiménez-Cauhe J, OrtegaQuijano D, Suárez-Valle A. Minor 
aphthae associated with SARSCoV-2 infection. Int $\mathrm{J}$ Dermatol. 2020;59(8):1022-3.

37. Gottlieb M, Long B. Dermatologic manifestations and complications of COVID-19. Am J Emerg Med. 2020;38(9):1715-21. https://doi.org/10 .1016/j.ajem.2020.06.011

38. Díaz Rodríguez M, Jiménez Romera A, Villarroel M. Oral manifestations associated with COVID-19. Oral Dis. 2020 Jul 22:10.1111/odi.13555. https:/ /doi.org/10.1111/odi.13555

39. Taşkın B, Vural S, Altuğ E, Demirkesen C, Kocatürk E, Çelebi, et al. Coronavirus 19 presenting with atypical Sweet's syndrome. J Eur Acad Dermatology Venereol. 2020;34(10):e534-5.

40. Putra BE, Adiarto S, Dewayanti SR, Juzar DA. Viral exanthem with "Spins and needles sensation" on extremities of a COVID-19 patient: a self-reported case from an Indonesian medical frontliner. Int J Infect Dis. 2020 Jul 1;96:355-8. https://doi.org/10. 1016/j.ijid.2020.05.020

41. Corchuelo J, Chavier F. Oral manifestations in a patient with a history of asymptomatic COVID-19: case report. Int $\mathrm{J}$ Infect Dis. 2020;100:154-7.

42. Jiménez-Cauhe J, Ortega-Quijano D, de Perosanz-Lobo D, BurgosBlasco P, Vañó-Galván S, FernándezGuarino $M$, et al. Enanthem in Patients With COVID-19 and skin rash. JAMA Dermatol. 2020 Jul 15; 156(10):1134-1136. https://doi.org/10. 1001/jamadermatol.2020.2550

43. Cruz Tapia RO, Peraza Labrador AJ, Guimaraes DM, Matos Valdez LH. Oral mucosal lesions in patients with SARS-CoV-2 infection. Report of four cases. Are they a true sign of
COVID-19 disease? Spec Care Dent. 2020;40(6):555-60.

44. Magro C, Mulvey JJ, Berlin D, Nuovo G, Salvatore S, Harp $\mathrm{J}$, et al. Complement associated microvascular injury and thrombosis in the pathogenesis of severe COVID-19 infection: a report of five cases. Transl Res. 2020;220:1-13. https://doi.org/10. 1016/j.trsl.2020.04.007

45. Cebeci Kahraman F, Çaşkurlu H. Mucosal involvement in a COVID-19. positive patient: a case report. Dermatol Ther. 2020 Jul;33(4):e13797. https://doi.org/10.1111/dth.13797

46. Navaeifar MR, Ghazaghi MP, Shahbaznejad L, Rouhanizadeh $\mathrm{H}$, Abutalebi M, Varandi MR, et al. Fever with rash is one of the first presentations of COVID-19 in children: a case report. Int Med Case Rep J. 2020;13:335-40.

47. Chiu JS, Lahoud-Rahme M, Schaffer D, Cohen A, SamuelsKalow M. Kawasaki disease features and myocarditis in a patient with COVID-19. Pediatr Cardiol. 2020;41(7):1526-8. https://doi.org/10. 1007/s00246-020-02393-0

48. Malih N, Hajinasrollah G, Zare M, Taheri M. Unexpected presentation of COVID-19 in a 38-year-old male patient: a case report. Case Rep Dermatol. 2020;12(2):124-31.

49. Tomo S, Miyahara GI, Simonato LE. Oral mucositis in a SARS-CoV-2infected patient: Secondary or truly associated condition? Oral Dis. 2020; Jul 29. https://doi.org/10.1111/odi.135 70

50. Olisova OY, Anpilogova EM, Shnakhova LM. Cutaneous manifestations in COVID-19: a skin rash in a child. Dermatol Ther. 2020 May:1-2. 
51. Madjid M, Safavi-Naeini P, Solomon SD, Vardeny O. Potential effects of coronaviruses on the cardiovascular system: a review. JAMA Cardiol. 2020 Jul;5(7):831-40.

52. Cebeci Kahraman F, Özen T, Elibol T. Lip necrosis in a patient with paroxysmal nocturnal hemoglobinuria: can it be triggered by COVID-19? J Cosmet Dermatol. 2020;19(12):3168-70. https://doi.org/1 0.1111 /jocd. 13746

53. Singh C, Tay J, Shoqirat N. Skin and mucosal damage in patients diagnosed with Covid-19 a case report. J Wound Ostomy Cont Nurs. 2020;47(5):435-8.

54. Mazzotta F, Troccoli T, Caselli D, Bonifazi E. Acral rash in a child with COVID-19. Eur J Pediatr Dermatology. 2020;30(2):79-82.

55. Labé P, Ly A, Sin C, Nasser M, Chapelon-Fromont E, Ben Saïd $\mathrm{P}$, et al. Erythema multiforme and Kawasaki disease associated with COVID-19 infection in children. J Eur Acad Dermatology Venereol. 2020;34(10):e539-41.

56. Farouk S, Sadek A. Cutaneous manifestations of COVID-19: a case report and a new finding from Egypt. Dermatol Ther. 2020;33(6):e14038. ht tps://doi.org/10.1111/dth.14038

57. Manzalawi R, Alhmamey K, Abdelrasoul M. Gingival bleeding associated with COVID-19 infection. Clin Case Reports. 2021;9(1):294-7.

58. Scully C, Samaranayake LP. Emerging and changing viral diseases in the new millennium. Oral Dis. 2016 Apr 1;22(3):171-9.

59. Kitakawa D, Oliveira FE, Neves De Castro P, Carvalho LFCS. Short report-Herpes simplex lesion in the lip semimucosa in a COVID-19 patient. Eur Rev Med Pharmacol Sci. 2020;24(17):9151-3.
60. Aghazadeh N, Homayouni M, Sartori-Valinotti JC. Oral vesicles and acral erythema: report of a cutaneous manifestation of COVID-19. Int J Dermatol. 2020;59(9):1153-4.

61. de Freitas Ferreira ACA, Romão TT, SIlva Macedo Y, Pupe C, Nascimento OJM. COVID-19 and herpes zoster co-infection presenting with trigeminal neuropathy. Eur J Neurol. 2020;27(9):1748-50. https://d oi.org/10.1111/ene.14361

62. Farabi B, Atak MF. Isolated maculopapular eruption localized to head and neck: a cutaneous sign of COVID-19 infection. Dermatol Ther. 2020;33(6):e14468. https://doi.org/10. 1111/dth.14468.

63. Riad A, Gad A, Hockova B, Klugar $M$. Oral candidiasis in non-severe COVID-19 patients: call for antibiotic stewardship. Oral Surg. 2020;1-2. http s://doi.org/10.1111/ors.12561

64. Salehi M, Ahmadikia K, Mahmoudi S, Kalantari S, Jamalimoghadamsiahkali S, Izadi A, et al. Oropharyngeal candidiasis in hospitalised COVID-19 patients from Iran: species identification and antifungal susceptibility pattern. Mycoses. 2020;63(8):771-8.

65. Zegarelli DJ. Fungal infections of the oral cavity. Otolaryngol Clin North Am. 1993 Dec;26(6):1069-89.

66. Pahl C, Yarrow S, Steventon N, Saeed NR, Dyar O. Angina bullosa haemorrhagica presenting as acute upper airway obstruction. BJA $\mathrm{Br}$ J Anaesth. 2004 Feb 1;92(2):283-6. http s://doi.org/10.1093/bja/aeh029

67. Jones VG, Mills M, Suarez D, Hogan CA, Yeh D, Bradley Segal J, et al. COVID-19 and Kawasaki Disease: Novel Virus and Novel Case. Hosp Pediatr. 2020;10(6). 
68. Jones I, Bell LCK, Manson JJ, Last A. An adult presentation consistent with PIMS-TS. Lancet Rheumatol. 2020 Jul;2 (9):e520-1. https://doi.org/1 0.1016/S2665-9913(20)30234-4

69. Estébanez A, Pérez-Santiago L, Silva Es, Guillen-Climent S, García- Vázquez A, Ramón MD, et al. Cutaneous manifestations in COVID-19: a new contribution. J Eur Acad Dermatology Venereol. 2020;34(6):e250-1.

70. Morey-Olivé M, Espiau M, Mercadal-Hally M, Lera-Carballo E, García-Patos V. Cutaneous manifestations in the current pandemic of coronavirus infection disease (COVID 2019). An Pediatr (Engl Ed). 2020;92(6):374-5.

71. Fernández Nieto D, Ortega Quijano D, Segurado Miravalles G, Pindado Ortega C, Prieto Barrios M, Jimenez Cauhe J, et al. Comment on: cutaneous manifestations in COVID-19: a first perspective. Safety concerns of clinical images and skin biopsies. J Eur Acad Dermatology Venereol. 2020;34(6):e252-4.

72. Henry D, Ackerman M, Sancelme E, Finon A, Esteve E. Urticarial eruption in COVID-19 infection. J Eur Acad Dermatology Venereol. 2020;34(6):e244-5.

73. Hedou M, Carsuzaa F, Chary E, Hainaut E, Cazenave-Roblot F, Masson Regnault M. Comment on 'Cutaneous manifestations in COVID-19: a first perspective' by Recalcati S. J Eur Acad Dermatology Venereol. 2020;34(7):e299-300.

74. van Damme C, Berlingin E, Saussez S, Accaputo O. Acute urticaria with pyrexia as the first manifestations of a COVID-19 infection. J Eur Acad Dermatology Venereol. 2020;34(7):e300-1.
75. Lu S, Lin J, Zhang Z, Xiao L, Jiang Z, Chen J, et al. Alert for non-respiratory symptoms of Coronavirus Disease 2019 (COVID-19) patients in epidemic period: A case report of familial cluster with three asymptomatic COVID-19 patients. J Med Virol. 2020; 2021 Jan;93(1):518-21. https://doi.org/10.10 02/jmv.25776

76. Rivera-Oyola R, Koschitzky M, Printy R, Liu S, Stanger R, Golant $\mathrm{AK}$, et al. Dermatologic findings in 2 patients with COVID-19. JAAD Case Rep. 2020;6(6):537-9. https://doi.org/1 0.1016/j.jdcr.2020.04.027

77. Sánchez A, Sohier P, Benghanem S, L'Honneur A-S, Rozenberg F, Dupin $\mathrm{N}$, et al. Digitate papulosquamous eruption associated with severe acute respiratory syndrome coronavirus 2 infection. JAMA Dermatol. 2020 Jul 1;156(7):819-20. https://doi.org/10.10 01/jamadermatol.2020.1704

78. Amatore F, Macagno N, Mailhe M, Demarez B, Gaudy-Marqueste C, Grob JJ, et al. SARS-CoV-2 infection presenting as a febrile rash. J Eur Acad Dermatology Venereol. 2020;34(7):e304-6.

79. Gisondi P, Piaserico S, Conti A, Naldi L. Dermatologists and SARSCoV-2: the impact of the pandemic on daily practice. J Eur Acad Dermatology Venereol. 2020;34(6):1196-201.

80. Pangti Rashi GS. Recognizable vascular skin manifestations of SARS CoV-2 infection are uncommon in patients with skin-of-color. Clin Exp Dermatol. 2021 Jan;46(1):180-182. ht tps://doi.org/10.1111/ced.14421

81. Recalcati S. Cutaneous manifestations in COVID-19: a first perspective. J Eur Acad Dermatology Venereol. 2020;34(5):e212-3. 
82. Kang JH. Febrile illness with skin rashes. Infect Chemother. 2015;47(3):155-66.

83. Ahouach B, Harent S, Ullmer A, Martres P, Bégon E, Blum L, et al. Cutaneous lesions in a patient with COVID-19: are they related? $\mathrm{Br} \mathrm{J}$ Dermatol. 2020;183(2):e31.

84. Mahé A, Birckel E, Merklen C, Lefèbvre P, Hannedouche C, Jost M, et al. Histology of skin lesions establishes that the vesicular rash associated with COVID-19 is not 'varicella-like.' J Eur Acad Dermatology Venereol. 2020;34(10):e559-61.

85. Hoenig LJ, Pereira FA. Eruption as a clinical manifestation of COVID-19: photographs of a patient. Clin Dermatol. 2020;38(4):502-5. https://doi.org/10.1 016/j.clindermatol.2020.04.001

86. Jiménez-Cauhe J, Ortega-Quijano D, Carretero-Barrio I, Suárez-Valle A, Saceda-Corralo D, Moreno-García del Real C, et al. Erythema multiforme-like eruption in patients with COVID-19 infection: clinical and histological findings. Clin Exp Dermatol. 2020;45(7):892-5.

87. Tatu AL, Nadasdy T, Bujoreanu FC. Familial clustering of COVID-19 skin manifestations. Dermatol Ther. 2020 Nov;33(6):e14181. https://doi.or g/10.1111/dth.14181

88. Rossi E, Lasagni C, Trakatelli M, Wertzberger Rowan S, Magnoni C. Acute maculopapular eruption in Covid-19 patient: A case report. Dermatol Ther. 2020 Nov;33(6):e13812. https://doi.org/10. 1111/dth.13812

89. Paolino G, Canti V, Mercuri SR, Rovere Querini P, Candiani M, Pasi F. Diffuse cutaneous manifestation in a new mother with COVID-19
(SARS-Cov-2). Int J Dermatol. 2020;59(7):874-5.

90. Robustelli Test E, Vezzoli P, Carugno A, Raponi F, Gianatti A, Rongioletti F, et al. Acute generalized exanthematous pustulosis with erythema multiforme-like lesions induced by Hydroxychloroquine in a woman with coronavirus disease 2019 (COVID-19). J Eur Acad Dermatology Venereol. 2020;34(9):e457-9.

91. Rosell-Díaz AM, Mateos-Mayo A, Nieto-Benito LM, Balaguer-Franch I, Hernández de la Torre-Ruiz E, Lainez-Nuez A, et al. Exanthema and eosinophilia in COVID-19 patients: has viral infection a role in drug induced exanthemas? J Eur Acad Dermatology Venereol. 2020;34(10):e561-3.

92. Danarti R, Budiarso A, Rini DLU, Soebono H. Follicular eruption as a cutaneous manifestation in COVID-19. BMJ Case Rep. 2020;13(10):e238182.

93. Tamai M, Maekawa A, Goto N, Ge L, Nishida T, Iwahashi $\mathrm{H}$, et al. Three cases of COVID-19 patients presenting with erythema. J Dermatol. 2020;47 (10):1175-8.

94. Sadr PZ, Pishbin E, Khodashahi R. A young woman with a full-body rash. J Am Coll Emerg Physicians Open. 2020;1(5):1145-6.

95. Lagziel T, Quiroga L, Ramos M, Hultman CS, Asif M. Two false negative test results in a symptomatic patient with a confirmed case of severe acute respiratory syndrome coronavirus-2 (SARSCoV-2) and Suspected StevensJohnson Syndrome/Toxic Epidermal Necrolysis (SJS/TEN). Cureus. 2020;12(5):e8198. https://doi.org/10.7 $759 /$ cureus. 8198 
96. Torres-Navarro I, AbrilPérez C, Roca-Ginés J, SánchezArráez J, Botella-Estrada R. A case of cefditoren-induced acute generalized exanthematous pustulosis during COVID-19 pandemics. Severe cutaneous adverse reactions are an issue. J Eur Acad Dermatology Venereol. 2020;34(10):e537-9.

97. Bursal Duramaz B, Yozgat CY, Yozgat Y, Turel O. Appearance of skin rash in pediatric patients with COVID-19: Three case presentations. Dermatol Ther. 2020;33(4):2-3.

98. Kaya G, Kaya A, Saurat J-H. Clinical and histopathological features and potential pathological mechanisms of skin lesions in COVID-19: review of the literature. Dermatopathology. 2020;7(1):3-16.

\section{Apéndice}

\section{Anexo}

Resumen de estudios relacionados con la asociación entre manifestaciones mucocutáneas orofaciales y la COVID-19

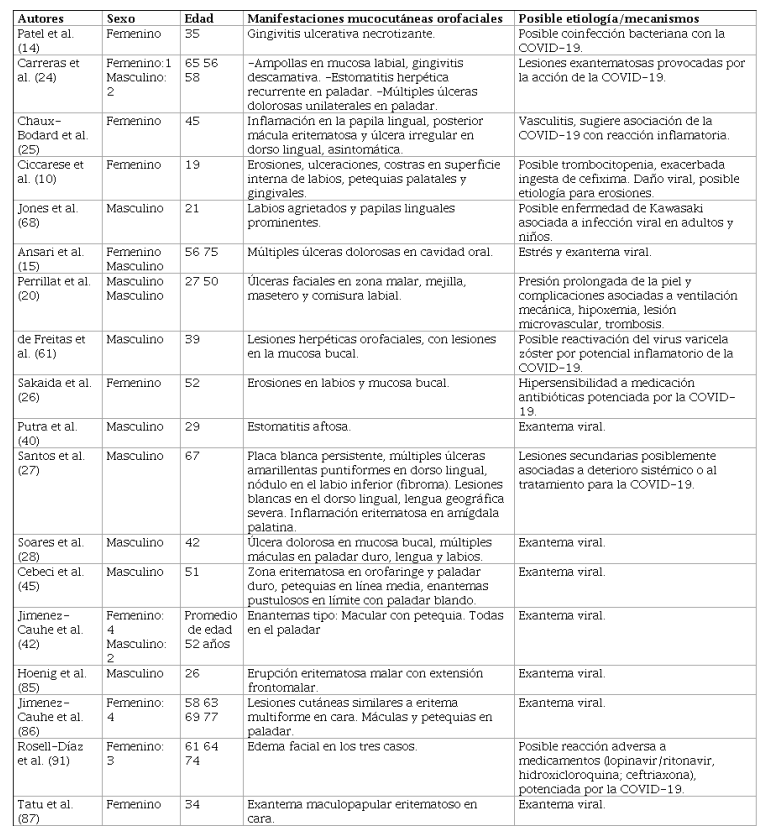

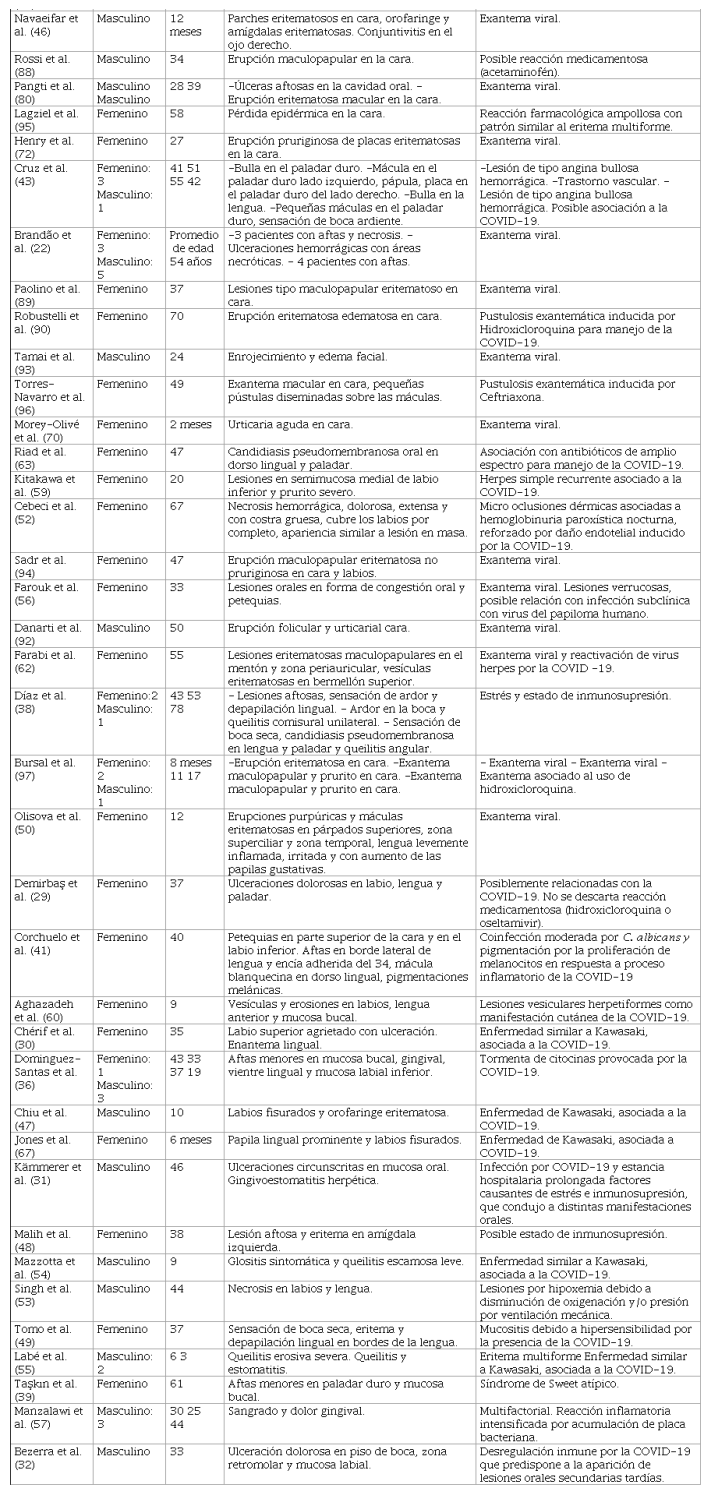

